\title{
Nonlinear Model Predictive Control of a Simplified Wind Turbine
}

\author{
Henriksen, Lars Christian; Poulsen, Niels Kjølstad; Hansen, Morten Hartvig
}

Published in:

Preprints of the 18th IFAC World Congress

Link to article, DOI:

10.3182/20110828-6-IT-1002.02070

Publication date:

2011

Document Version

Publisher's PDF, also known as Version of record

Link back to DTU Orbit

Citation (APA):

Henriksen, L. C., Poulsen, N. K., \& Hansen, M. H. (2011). Nonlinear Model Predictive Control of a Simplified Wind Turbine. In Preprints of the 18th IFAC World Congress (pp. 551-556). International Federation of Automatic Control. https://doi.org/10.3182/20110828-6-IT-1002.02070

\section{General rights}

Copyright and moral rights for the publications made accessible in the public portal are retained by the authors and/or other copyright owners and it is a condition of accessing publications that users recognise and abide by the legal requirements associated with these rights.

- Users may download and print one copy of any publication from the public portal for the purpose of private study or research.

- You may not further distribute the material or use it for any profit-making activity or commercial gain

- You may freely distribute the URL identifying the publication in the public portal 


\title{
Nonlinear Model Predictive Control of a Simplified Wind Turbine
}

\author{
L. C. Henriksen ${ }^{*}$ N. K. Poulsen ${ }^{* *}$ M. H. Hansen ${ }^{* * *}$ \\ * Wind Energy Division, Risø National Laboratory for Sustainable \\ Energy, Technical University of Denmark, larh@risoe.dtu.dk \\ ** Dept. of Informatics and Mathematical Modelling, Technical \\ University of Denmark, nkp@imm.dtu.dk \\ *** Wind Energy Division, Risø National Laboratory for Sustainable \\ Energy, Technical University of Denmark, mhha@risoe.dtu.dk
}

\begin{abstract}
This paper discusses the implications of formulating a single control law governing the entire wind speed range of operation for a wind turbine. Furthermore, the knowledge of future wind speeds provided by e.g. LIDARs is included in the controller framework. This is possible as the presented controller is based on nonlinear model predictive control and includes the knowledge of the future wind speed in the prediction horizon of the controller. The potential benefits of exploiting the knowledge provided by LIDARs is demonstrated in simulations with a simplified 1 degree-of-freedom nonlinear wind turbine model.
\end{abstract}

Keywords: nonlinear model predictive control, constraint handling, horizontal axis wind turbine, lidar

\section{INTRODUCTION}

The variable-speed horizontal-axis pitch-controlled wind turbine [Burton et al., 2001] pose some interesting challenges with respect to the design of controllers. Partial load wind speeds, that is wind speeds where the power produced by the wind turbine is below the nominal generator power, the wind turbine controller seeks to maximize the wind power converted by the wind turbine. In this operating regime the wind turbine dynamics are highly nonlinear as the wind turbine operates on the top of the power coefficient $\left(C_{P}\right)$ curve and depending on which side of the $C_{P}$ curve the wind turbine currently operates the aerodynamic gradients switches sign when going from one side of the $C_{P}$ curve to the other. For above rated wind speeds, the wind turbine has reached its nominal generator power and has pitched away from the top of the $C_{P}$ curve. On the slope of the $C_{P}$ curve, the wind turbine dynamics can be considered linear for reasonable small perturbations around the current operating point. Controllers usually switch between partial and full load configurations and the two configurations are usually quite different, where virtually no pitch action occurs for partial load operation and the electromagnetic generator torque attempts to keep the wind turbine at an optimal point of power capture, the roles reverse for full load operation where the generator torque action is reduced and the pitch control takes over.

There are good reasons to use pitch control below rated wind speeds as the spatial distribution of wind speeds seen in the area of the rotor disc is not uniform nor constant and load reductions on the key structures can be achieved by prober pitch action. Wake meandering, tower shadow and wind shear effects calls for individual pitch action, a subject not investigated further in this work. Another type of disturbance is rotor-wide wind speed changes, such as a extreme operating gust (EOG) [IEC/TC88, 2005], disturbances of that nature are the subject of interest for this work. The knowledge of future changes in wind speed can e.g. by achieved by the use of lidars [Angelou et al., 2010], by upstream meteorology masts or wind speed estimations of upstream wind turbines in a wind farm.

Nonlinear model predictive control (NMPC) [Qin and Badgwell, 2003] fit the needs for control of wind turbines with the knowledge of future wind speeds. NMPC is able to cope with the nonlinearities caused by operation below rated speeds and the knowledge of future wind speeds can be included in the prediction horizon of the NMPC. For wind speeds clearly in the partial or full load regions the NMPC controller can operate with a control law designed specifically for the particular region of operation. For wind speeds just around rated wind speed, the need for a single a control law is apparent. Initial thoughts of how to device such a control law are presented in this paper. NMPC has previously been applied for wind turbine control [Trainelli et al., 2006, Santos, 2007], but no special attention has been brought on below rated operation and on the switching between regions of operation.

The NMPC algorithm used in this work [Henriksen and Poulsen, 2010b,a] is partly based on the work of Tenny et al. [2004] and Rao et al. [1998] where the special structure of the MPC is exploited to reduce the computational burden of NMPC with long prediction horizons.

This paper is composed in the following way: The wind turbine model is presented first. The wind turbine model is followed by a section describing NMPC in general terms. The third section discusses relevant objectives for the wind turbine controller and discusses different options for implementing a single full wind speed range control law. 
Finally results of simulations with the NMPC for above and below rated are presented and conclusions are made.

\section{WIND TURBINE MODEL}

The wind turbine used in this work is a 1 degree of freedom model with physical parameters similar to those of the NREL 5MW reference wind turbine defined by Jonkman et al. [2009]. A wind turbine of this type is controlled by the pitch angle $\theta$ of the blades and the electromagnetic torque of the generator $Q_{g}$, where generator torque in the one end of the drivetrain and aerodynamic rotor torque $Q$ in the other determines whether the wind turbine rotational speed $\Omega$ is increased or decreased. The state space ordinary differential equation for the simplified wind turbine model, augmented with integrators between the control signals $u_{1}$ and $u_{2}$ and the pitch angle and generator torque, is

$$
\left[\begin{array}{c}
\dot{\Omega} \\
\dot{\theta} \\
\dot{Q}_{g}
\end{array}\right]=\left[\begin{array}{c}
\frac{1}{J_{t}}\left(Q(V, \Omega, \theta)-Q_{g}\right) \\
u_{1} \\
u_{2}
\end{array}\right]
$$

with the aerodynamic torque $Q$ given by

$$
Q(V, \Omega, \theta)=\frac{\frac{1}{2} \rho \pi R^{2} V^{3} C_{P}(V, \Omega, \theta)}{\Omega}
$$

where $\rho$ is mass density of air, $R$ is the rotor radius and $C_{P}$ is the power coefficient describing how much of the power available in the air is captured by the rotor. Generator power is given by

$$
P_{e}=Q_{g} \Omega_{g} \eta, \quad \Omega_{g}=\Omega N_{g}
$$

where $\eta$ is the efficiency factor describing losses in gear, power electronics etc and $N_{g}$ is the gear ratio. The aerodynamic power coefficient $C_{P}$ can be mapped to be a function of $(\lambda, \theta)$ rather than $(V, \Omega, \theta)$ where $\lambda=\Omega R / V$ as seen in Fig. 1(a).

The augmentation of the model, given by (1), with the two integrators is done to enable constraints on the control signal rates as well as on the control signals themselves.

\section{NONLINEAR MODEL PREDICTIVE CONTROL}

In this section the concept of Nonlinear Model Predictive Control will be presented. NMPC predicts the future behavior of the plant based on the model, current state estimates, and available knowledge of controlled and uncontrolled inputs. Controlled inputs are in this case the pitch angle and generator torque and the uncontrolled input is the wind speed. Typically no future knowledge of the disturbances is available, but with the naive assumption that e.g. a LIDAR is able to provide information about future wind speeds, this information can be used by the NMPC algorithm.

\subsection{Constrained Dynamic Optimization}

The control law is given by the minimization of the cost function $\Phi$ from current time $t$ to the end of the prediction horizon at time $t+t_{f}$ with $N+1$ discrete points within the finite prediction horizon

$$
\min \Phi_{N}\left(\boldsymbol{x}_{N}\right)+\sum_{k=0}^{N-1} \Phi_{k}\left(\boldsymbol{x}_{k}, \boldsymbol{u}_{k}, \boldsymbol{s}_{k}, \boldsymbol{r}_{k}\right)
$$

subject to the following inequality constraints (4c) to (4e) and state progress constraint (4b)

$$
\begin{aligned}
\underline{\boldsymbol{f}}\left(\boldsymbol{x}_{k}, \boldsymbol{u}_{k}, \boldsymbol{d}_{k}\right)-\boldsymbol{x}_{k+1} & =\mathbf{0} \\
\boldsymbol{c}_{h}\left(\boldsymbol{x}_{k}, \boldsymbol{u}_{k}\right) & \leq \mathbf{0} \\
\boldsymbol{c}_{s}\left(\boldsymbol{x}_{k}, \boldsymbol{u}_{k}\right)-\boldsymbol{s}_{k} & \leq \mathbf{0} \\
\boldsymbol{s}_{k} & \geq \mathbf{0}
\end{aligned}
$$

where $\boldsymbol{x}$ are states, $\boldsymbol{u}$ are controlled inputs, $\boldsymbol{d}$ are uncontrolled inputs or disturbances, $\boldsymbol{r}$ are the references to be tracked and $s$ are the slack variables associated with the soft constraint inequality. $\boldsymbol{f}(\cdot)$ is the state progress equation, $\boldsymbol{c}_{\boldsymbol{h}}(\cdot)$ is the hard constraints inequality and $\boldsymbol{c}_{\boldsymbol{s}}(\cdot)$ is the soft constraints inequality. The total stage-wise cost function $\Phi_{k}\left(\boldsymbol{x}_{k}, \boldsymbol{u}_{k}, \boldsymbol{s}_{k}, \boldsymbol{r}_{k}\right)$ is the sum of the reference tracking cost (power control)

$$
\Phi_{r}\left(\boldsymbol{x}_{k}, \boldsymbol{u}_{k}, \boldsymbol{r}_{k}\right)=\left\|\boldsymbol{y}\left(\boldsymbol{x}_{k}, \boldsymbol{u}_{k}\right)-\boldsymbol{r}_{k}\right\|_{\mathbf{W}_{\mathbf{r}}}^{2}
$$

the dynamic cost (load reduction)

$$
\Phi_{z}\left(\boldsymbol{x}_{k}, \boldsymbol{u}_{k}\right)=\left\|\boldsymbol{z}\left(\boldsymbol{x}_{k}, \boldsymbol{u}_{k}\right)\right\|_{\mathbf{W}_{\mathbf{z}}}^{2}
$$

and of the cost of violating soft constraints

$$
\Phi_{s}\left(s_{k}\right)=\left\|s_{k}\right\|_{\mathbf{W}_{\mathrm{s}}}^{2}
$$

A terminal cost $\Phi_{N}\left(\boldsymbol{x}_{N}\right)$ can be appended to achieve closed loop stability [Chen and Allgower, 1998]. But as discussed later on, this is not possible for the below rated operation and has thus been omitted from the controller. The open-loop system is not always stable and closedloop predictions could be used to stabilize the system predictions within the prediction horizon [Tenny et al., 2004]. Open-loop instabilities have not given cause to any concern in the results obtained so far and has therefore not been implemented in the present work.

\subsection{Time-discretization: Non-equidistant spacing}

To exploit the potential benefit of having the knowledge of future wind speeds obtained by e.g. LIDARs, prediction horizons matching the length of future available knowledge are needed. It becomes beneficial w.r.t. computational costs to reduce the number of time-discrete points in the prediction horizon for long prediction horizons. The reduction of temporal points can be achieved by having a fine temporal resolution in the beginning of the prediction horizon and a coarser resolution towards the end of the prediction horizon. A non-equidistant temporal distribution means that the cost function can no longer be considered the sum of a number of equally important cost and the cost at a given temporal point should be weighted according to the current temporal spacing. Trapezoidal integration of the costs w.r.t. the temporal points of the prediction horizon ensures a proper weighting of the different cost along the prediction horizon. The temporal integration of the state progress equation should also be done with the non-equidistant temporal spacing in mind. Time discretization of the state progress equation can be done with e.g. forward Euler, Runge-Kutta schemes such as ESDIRK Kristensen et al. [2004], collocation points Biegler [2007] etc. The nonlinear model within the NMPC algorithm is repeatedly called throughout the temporal integration within a time step, resulting in a lot of computationally expensive function calls. To ease the computational burden, the model can be assumed to be linear within a time step and time integration can be performed using zeroorder-hold or linear forward Euler. For larger time steps 
as seen in the end of the prediction horizon zero-order-hold did not give satisfactory results and forward Euler has as result been used throughout this work. The assumption of linear dynamics within a time step might lead to poor convergence as the dynamics might change a lot from one iteration to another, especially for large time steps, it remains to be investigated whether the assumption of linear dynamics within a time step leads to overall faster computations or not.

\subsection{Steady state performance}

One method to determine if the control objectives achieve the desired steady state for a given constant wind speed is to perform an optimization with a cost function as one of the stage-wise costs from the dynamic optimization

$$
\min _{\overline{\boldsymbol{x}}, \overline{\boldsymbol{u}}, \overline{\boldsymbol{s}}} \Phi_{t o t}(\overline{\boldsymbol{x}}, \overline{\boldsymbol{u}}, \overline{\boldsymbol{s}}, \overline{\boldsymbol{r}})
$$

s.t.

$$
\begin{aligned}
\boldsymbol{c}_{h}(\overline{\boldsymbol{x}}, \overline{\boldsymbol{u}}) & \leq \mathbf{0} \\
\boldsymbol{c}_{s}(\overline{\boldsymbol{x}}, \overline{\boldsymbol{u}})-\boldsymbol{s} & \leq \mathbf{0} \\
\boldsymbol{f}(\overline{\boldsymbol{x}}, \overline{\boldsymbol{u}}) & =\mathbf{0}
\end{aligned}
$$

where the prediction horizon has been omitted from the optimization and the state progress equation has been replaced with the ordinary differential equation for the model.

\subsection{Optimization algorithm}

A trust-region-based sequential quadratic programming algorithm Henriksen and Poulsen [2010b] framework has been used as the optimization algorithm for both the steady state and for the dynamic optimization problems, where the underlying quadratic programming problem (QP) solvers, quasi-Newton Hessian approximations and trust-regions are specifically tailored for the specific problem. The steady state optimization uses a general purpose QP solver and produces dense quasi-Newton Hessian approximations and trust-regions. The dynamic optimization on the other hand use a QP solver exploiting the structure of the problem [Rao et al., 1998, Henriksen and Poulsen, 2010a] and quasi-Newton Hessian approximations and trust-regions also exploit the special structure of the problem as described by Tenny et al. [2004].

\section{STEADY STATE CONTROL OBJECTIVES FOR THE WIND TURBINE}

The first and foremost objective of the wind turbine control is to produce as much power as possible below rated wind speeds and to produce the nominal power above rated wind speeds. This objective can be formulated as

$$
J=\left(P_{e}-P_{n o m}\right)^{2}+w\left(\Omega_{g}-\Omega_{g, n o m}\right)^{2}
$$

where the additional term concerning generator speed $\Omega_{g}$ is only active for above rated wind speeds. This leads to $w=0$ for partial load and $w=1$ for full load. If the second term was omitted, then the generator speed could be a number of different values which together with a number of different pitch angles all lie on the same contour of the $C_{P}$ curve. A soft constraint on generator speed, confining the generator speed to its allowed operating range, is active for $w=0$. For $w=1$ the second term is expected to keep generator speed within the allowed operating range.

A sweep of wind speeds ranging from $V_{\text {high }}(=25 \mathrm{~m} / \mathrm{s})$ to $V_{\text {low }}(=3 \mathrm{~m} / \mathrm{s})$ is investigated for steady state values. The sweep is started at the highest wind speed $V_{\text {high }}$ and the initial guess is ensured to be on the pitch side of the $C_{P^{-}}$ curve. As steady state values for the initial wind speed is determined the second highest wind speed is investigated with the optimal values of the higher wind speed. Once partial load wind speeds are reached, the optimization is no longer able to determine values which ensure a power production at nominal power. The operating region weight $w$ is changed to partial load and the sweep is continued for the below rated wind speeds. The mixed integer switching between one region of operation and another is not suitable for the dynamic optimization where regions of operation switch inside the prediction horizon. A smoother weight $w$ depending on e.g. generator power should be designed and gradients for the gradients of $w$ should be included in the gradients provided to optimization solver.

If the wind sweep goes from low to high wind speeds, a closer look at Fig. 1(a) shows that up until rated wind speed the wind turbine is operating on the ridge of the $C_{P}$ curve. As rated wind speed is reached, power should be reduced and one of two options should taken: Either pitch to the stall side of the $C_{P}$ curve or to the pitch side. The optimization algorithm has no preference to which option to choose and should be aided to ensure that solutions are found on the pitch side of the $C_{P}$ curve rather than on the stall side. Nonlinear constraints could be imposed ensuring only feasible solutions on the pitch side another option is to use the fact that the optimization algorithm is gradient based and the aerodynamic gradient w.r.t. to pitch can be truncated such that increase in pitch angle always lead to decrease in $C_{P}$.

\section{RESULTS}

In this section results for the NMPC applied on a simplified wind turbine model is presented. First, partial load operation is examined and afterwards performance of full load operation is investigated. Two configurations of the NMPC is used: The first, NMPC ${ }^{\text {lidar }}$, assumes that knowledge of future wind speeds is available within the entire prediction horizon. Whereas the second, $\mathrm{NMPC}^{\text {normal }}$, has the same prediction horizon length as NMPClidar but assumes the currently measured/estimated wind speed to remain constant throughout the prediction horizon.

\subsection{Partial load operation}

In this section partial load performance is investigated. No simulation results are presented as computation times where to long, only the finally iterated prediction horizons of the two controller configurations are shown in Fig. 2. The prediction horizon is extremely long, in this case 250 s. The necessity for a long prediction horizon can be seen by examination of the plots in Fig. 2 in the last approx. 50 $\mathrm{s}$ of the prediction horizon generator power is increased at the expense of generator speed. This behavior is as prescribed by the cost function (9) and to achieve good closed loop control the prediction horizon should be so 
(Solid) $\left(\theta^{*}(V), \lambda^{*}(V)\right) \quad$ (Dashed) $\nabla_{\theta} C_{P}(\lambda)$

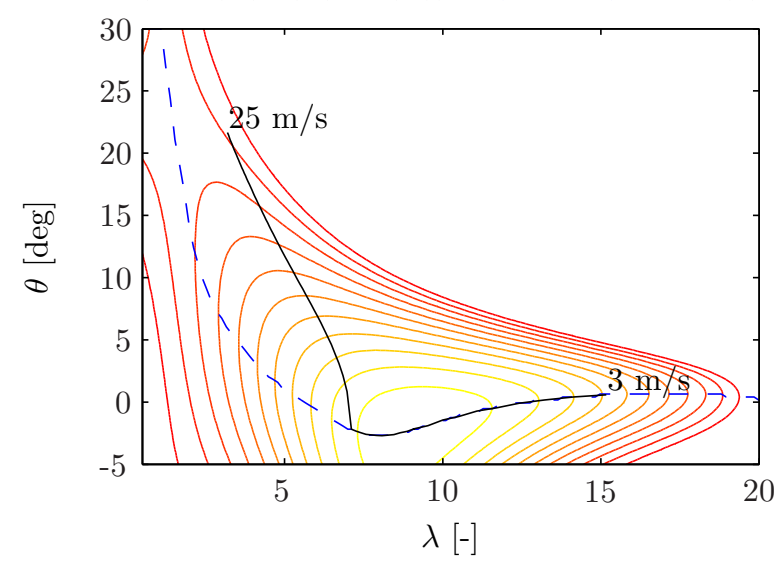

(a) $C_{P}$-curve
$(-) P_{e} \quad(-) \Omega(-) \theta$

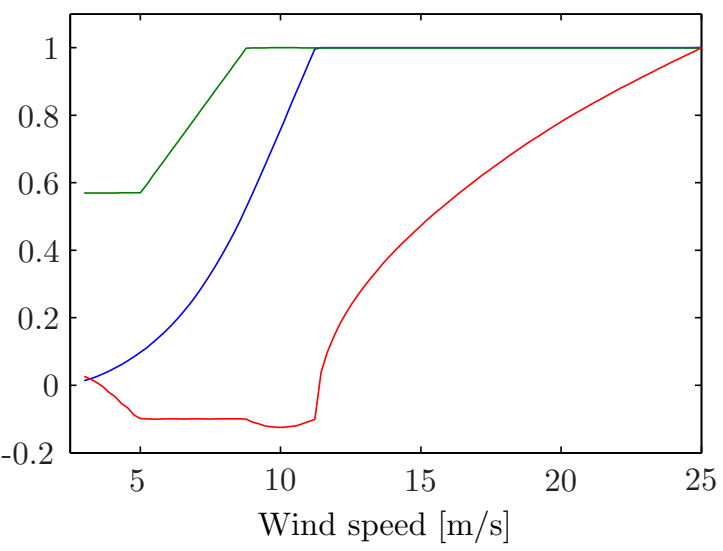

(b) Primary variables

Fig. 1. Wind sweep for steady state operating points of the wind turbine: Fig. 1 (a) depicts the $C_{P}$-curve and shows the steady state pitch angles and tip-speed-ratios for a sweep of wind speeds. Fig. 1(b) plots the normalized state values of generator power, generator speed and pitch angle.

long that the last $50 \mathrm{~s}$ does not influence the beginning of the prediction horizon. Another solution would be to append a suitable infinite horizon control law in the form of a terminal cost [Chen and Allgower, 1998]. The problem would however not be solved by appending a linear control law as the wind turbine is operating on the top of the $C_{P}$ curve in partial load where the temporal dynamics of the wind turbine are approximated better by second order Taylor expanded models than by first order Taylor expanded models. Another option would be to change the control objectives in partial load operation from optimization of generator power to optimization of $C_{P}$, but different control objectives for partial and full load operation might lead to problems regarding convergence of the dynamic optimization problem when switching between the operating regions occur within the prediction horizon.

Another interesting observation, unfortunately not easily seen in Fig. 2, is that for the NMPC ${ }^{\text {lidar }}$ the wind turbine is accelerated to higher rotational speed prior to the EOG. This is done to optimize the cost function and to produce more power than would have been produced if the rotational speed had not been accelerated. This observation indicates that also for more complex wind structures e.g. turbulence, the wind turbine would be able to produce more power at partial load operation if information of future wind speeds where available and if these predicted wind speeds where to be trusted.

\subsection{Full load operation}

In this section, results for the performance of the NMPC for full load operation is presented. An extreme operating gust occurs and as time progresses NMPClidar the EOG moves into the prediction horizon of NMPC ${ }^{\text {lidar }}$ and future control moves are planned accordingly, the outcome of the two controller configurations can be seen in Fig. 3. The pitch action of the two controllers is quite similar as shown in Fig. 3(b) but the resulting generator speed is better controlled when the information of future wind speed is included in the prediction horizon. The better controlled generator speed can be seen as an indication of the load reduction potential when applied on wind turbine models with more degrees of freedom.

\section{CONCLUSION AND FUTURE WORK}

A Nonlinear Model Predictive Control algorithm has been presented and its application on a wind turbine has been shown. Initial thought concerning the implementation of a full wind speed range control law has been expressed and results for the implemented controller has been shown both in partial and full load operation. It has been shown that inclusion of future wind speeds in the prediction horizon is beneficial and leads to increased power production for partial load operation and to decreased loads for full load operation.

It remains to be determined how to achieve good combined full and partial load operation within the prediction horizon. Different strategies for operating region switching exist: The operating region weight $w$ could be made continuous and dependant of e.g. $P_{e}$ in a smooth way to aid the optimization algorithm, that way the operating regions would not be clearly defined within the prediction horizon and as the optimization routine iterates the regions of operation change according to the current iterate, which might leave the optimization in limbo as the cost function is not well defined and convex. Another measure for determining which operating region should be active at a given point in time within the prediction horizon could be to make $w$ dependant of the predicted wind speed for the given point in time in the prediction horizon, that way the iterations would not change $w$ and the cost function would remain constant and better convergence properties might be achieved. Yet another option would be to make the region of operation dependant of a discrete set of rules for but as for the first option this would mean that the cost function could change from one iteration to the other and lead to poor convergence properties of the optimization algorithm. Future work should investigate these details. 
(Solid) NMPC lidar (Dashed) NMPC ${ }^{\text {normal }}$
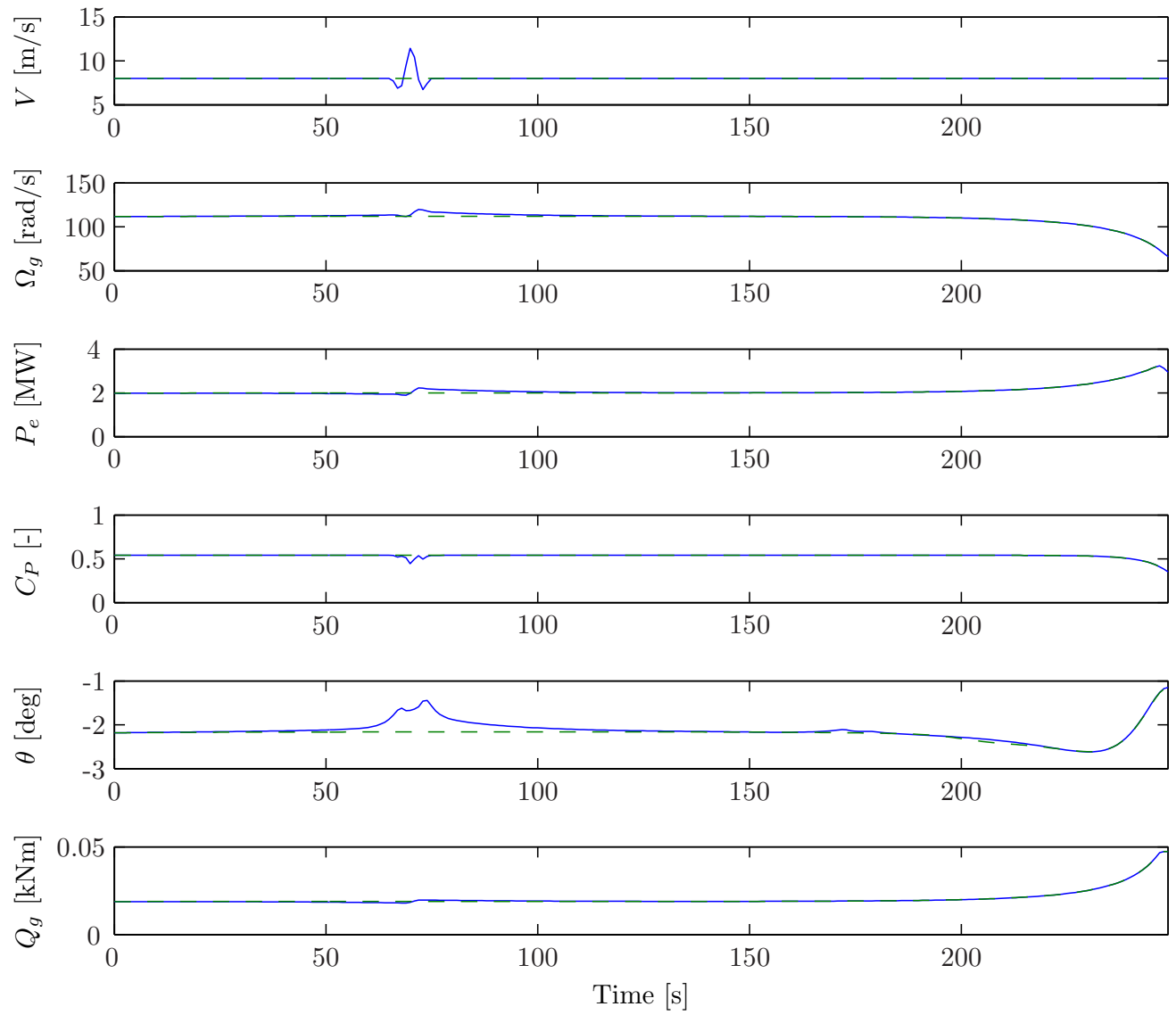

Fig. 2. Partial load operation: Prediction horizon of NMPC with no terminal cost. The plots show the predictions made by the controller at a given point in time.

\section{BIBLIOGRAPHY}

N. Angelou, T. Mikkelsen, K. H. Hansen, M. Sjöholm, and M. Harris. Lidar wind speed measurements from a rotating spinner: "spinnerex 2009". Ris $\varnothing-\mathrm{R} 1741$, Ris $\varnothing$ National Laboratory for Sustainable Energy, Technical University of Denmark, 2010.

L. T. Biegler. An overview of simultaneous strategies for dynamic optimization. Chem. Eng. Process.: Process Intensif., 46(11):1043-1053, 2007.

T. Burton, D. Sharpe, N. Jenkins, and E. Bossanyi. Wind Energy Handbook. John Wiley And Sons Ltd, 2001.

H. Chen and F. Allgower. Quasi-infinite horizon nonlinear model predictive control scheme with guaranteed stability. Automatica, 34(10):1205-1217, 1998.

L. C. Henriksen and N. K. Poulsen. An online relinearization scheme suited for model predictive or linear quadratic control. IMM-Technical Report 201013, Dept. of Informatics and Mathematical Modelling, Technical University of Denmark, 2010a.

L. C. Henriksen and N. K. Poulsen. A trust-region-based sequential quadratic programming algorithm. IMMTechnical Report 2010-14, Dept. of Informatics and Mathematical Modelling, Technical University of Denmark, 2010b.

IEC/TC88. IEC 61400-1 Ed.3: Wind turbines - Part 1: Design requirements. International Electrotechnical Commission (IEC), 82005.
J. Jonkman, S. Butterfield, W. Musial, and G. Scott. Definition of a 5 -mw reference wind turbine for offshore system development. Technical Report NREL/TP-50038060, National Renewable Energy Laboratory, 1617 Cole Boulevard, Golden, Colorado 80401-3393, February 2009 .

M. R. Kristensen, J. B. Jørgensen, P. G. Thomsen, and S. B. Jørgensen. An esdirk method with sensitivity analysis capabilities. Comput. Chem. Eng., 28(12): 2695-2707, 2004.

S. J. Qin and T. A. Badgwell. A survey of industrial model predictive control technology. Control Engineering Practice, 11(7):733-764, 2003.

C. V. Rao, S. J. Wright, and J. B. Rawlings. Application of interior-point methods to model predictive control. $J$. Optim. Theory Appl. (USA), 99(3):723-757, 1998.

R. Santos. Damage mitigating control for wind turbines. $\mathrm{PhD}$ thesis, University of Colorado at Boulder, United States - Colorado, 2007.

M. J. Tenny, S. J. Wright, and J. B. Rawlings. Nonlinear model predictive control via feasibility-perturbed sequential quadratic programming. Computational $\mathrm{Op}$ timization and Applications, 28(1):87-121, 2004.

L. Trainelli, W. Sirchi, B. Savini, A. Croce, and C. L. Bottasso. Aero-servo-elastic modeling and control of wind turbines using finite-element multibody procedures. Multibody System Dynamics, 16(3):291-308, 2006 . 
(Solid) NMPC ${ }^{\text {lidar }}$ (Dashed) NMPC normal
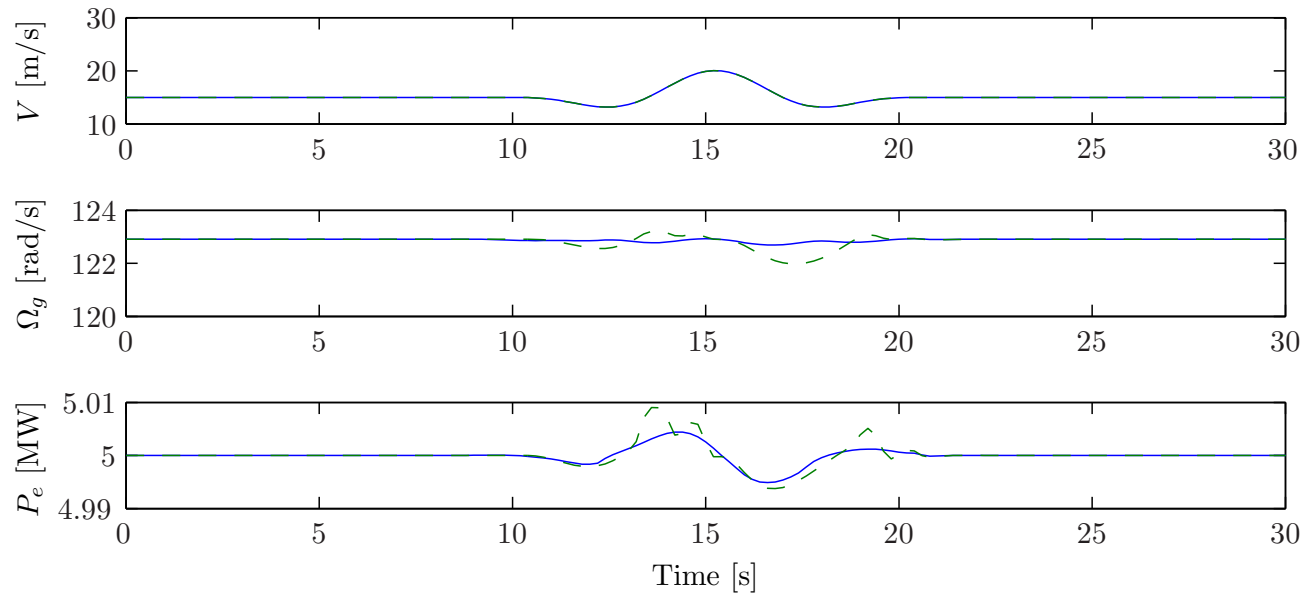

(a) Wind speed, generator speed and power.

(Solid) NMPC lidar $\quad$ (Dashed) NMPC normal
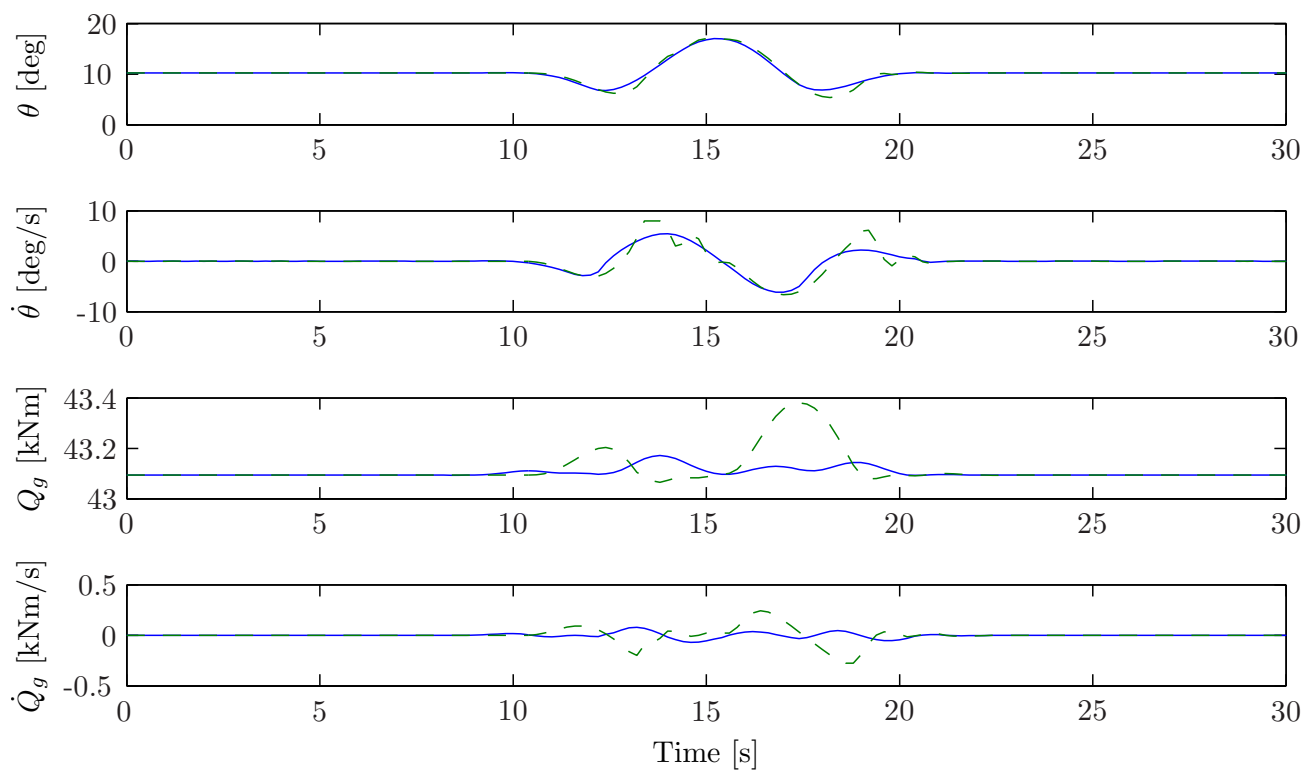

(b) Controlled inputs to plant: Pitch angle and rate, and generator torque and rate.

Fig. 3. Above rated wind speed: Simulations where an extreme operating gust occurs. Two nonlinear model predictive controllers are compared: The first, $\mathrm{NMPC}^{\text {lidar }}$, includes knowledge of future wind speeds in the prediction horizon. The second, NMPC ${ }^{\text {normal }}$, assumes that the current wind speed remains constant throughout the prediction horizon. 